\title{
New Pharmacologic Perspectives in Pneumology: Beclomethasone-Formoterol Extrafine
}

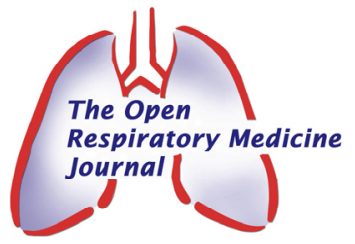

\author{
Pierluigi Paggiaro*
}

\author{
Cardio-Thoracic and Vascular Department, University of Pisa, Italy
}

\begin{abstract}
International asthma guidelines have recently focused on the concept of «control», which is the main outcome to reach and maintain in the long term management. Asthma control is associated with several positive consequences, both in terms of quality of life and pathophysiological findings. Combination therapy with inhaled corticosteroids (ICS) and long-acting-beta2-agonists (LABA) is recommended in a large part of asthmatic subjects (those who are not controlled with low-dose ICS alone).

Recently, a new beclomethasone/formoterol combination in an extrafine HFA formulation has been developed. This new technology allows to obtain a very high fine particle fraction which reaches lower airways, while the dose which remain in the upper airways and possibly responsible for systemic side effects is very low. Therefore, this combination allows a different dose ratio between BDP and the other ICS (budesonide, fluticasone), in favour of a lower dose of BDP. Recent studies have demonstrated the equivalence of this new combination with the other ICS/LABA combination, as regards all asthma outcomes. Then, this new BDP/formoterol combination may increase the possibility to manage adequately patients with moderate-to-severe asthma.
\end{abstract}

Keywords: Asthma, control, combination therapy, beclomethasone, formoterol.

\section{INTRODUCTION}

Despite the great work performed in the last years in the dissemination and implementation of asthma guidelines, the control of asthma in the world and in Europe is still poor. Many surveys have been conducted on this point, showing that just a minority of asthmatics, particularly between subjects with the greater level of severity, reach a good control of asthma [1]. There are several reasons for this inadequate management of asthma, including the underdiagnosis of the disease, the lack of appropriate pharmacologic strategies, and the low use of non pharmacologic strategies for the management of the disease (education, prevention, and adequate follow-up).

More recent guidelines have considered this problem, and they have simplified the management of asthma in order to have a better adherence of doctors and patients to the optimal strategy [2].

\section{THE VALUE OF THE “CONTROL” OF ASTHMA}

The aim of the management of asthma is now to reach and maintain the control of the disease. Considering that more ambitious aims (like the "cure" of asthma or the change in the natural history of the disease) are not realistic, the main outcome is to reach an almost complete remission of the disease, evaluated as the almost complete absence of daytime and nocturnal symptoms, rescue medication use and exacerbations, as well as a normal lung function and the absence of limitation in the daily life. This aim can be obtained

*Address correspondence to this author at the Dipartimento Cardio-Toracico e Vascolare, Ospedale di Cisanello, Via Paradisa 2, 56100 Pisa, Italy;

Tel: +39-050-995366; Fax: +39-050-580124;

E-mail: lpaggiaro@dcap.med.unipi.it in a majority of asthmatic patients with an appropriate treatment strategy, as demonstrated by the GOAL study [3].

The control of asthma is defined according to the criteria reported in Fig. (1). With an integrated evaluation of symptoms, use of rescue medication, level of pulmonary function, and rate of exacerbations, the control of the disease can be evaluated in a fairly easy way. The assessment of the control is necessary for the decision about the asthma management: the lack of control requires immediately a change in the level of pharmacologic treatment and a revision of the management plan, while a good control lead to consider the persistence or the step-down in the level of asthma treatment. This method of the evaluation of the control is simple and easily performed by the patient and the doctor, and this may improve the global management of the disease.

Reaching the control has positive consequences on the quality of life of the asthmatic patients [4], and reflects also in a progressive improvement of the indirect markers of airway inflammation [5].

\section{STRATEGIES TO OBTAIN AND MAINTAIN THE CONTROL}

Regular treatment with inhaled corticosteroids (ICS) is the cornerstone of asthma management. Some patients may reach the control with a monotherapy with low-dose ICS, but in a large part of asthmatic subjects this is not possible. In these cases, the best option is to use a combination with ICS and long-acting-beta2-agonists (LABA). Combination therapy has been demonstrated able to obtain the control of asthma better than ICS alone [3,6]. This fact has been related to the complementary effect of ICS and LABA on the different components of the disease (inflammation and smooth muscle disfunction) and in part also to some 


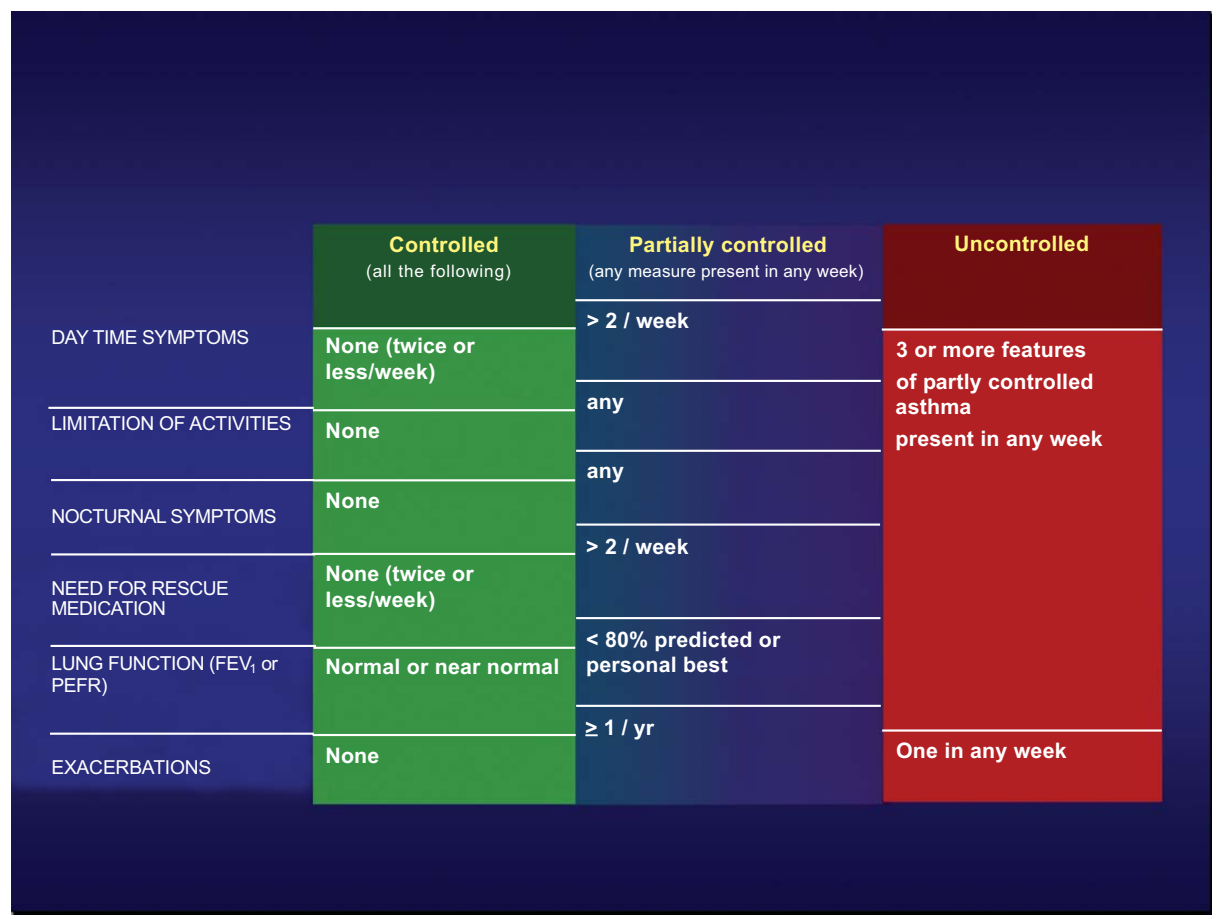

Fig. (1). Definition of the different levels of asthma control according to International GINA Guidelines [2].

synergistic effect [7]. For these reasons, combination therapy is recommended as a first line therapy for moderate-tosevere asthmatic patients $[1,8,9]$.

Two ICS-LABA combinations have been studied in the last 10 years: salmeterol/fluticasone and formoterol/budesonide. Few comparative studies have been reported, but a recent metanalysis suggests a substantial equivalence of these two combinations [10].

\section{A NEW FIXED ICS-LABA COMBINATION}

Recently a new fixed ICS-LABA combination has been developed and it is currently on the market in many European countries. This combination includes beclomethasone propionate (100 mcg per each inhalation) and formoterol (6 mcg per each inhalation). The main feature of this combination lays in the innovative formulation characterised by an extrafine particle size of drug particles. In fact, both BDP and formoterol display a mass median aerodynamic diameter (MMAD) in the range of 1.4-1.5 $\mu \mathrm{m}$. Consequently, the amount of drug delivered to the lung, and responsible for the therapeutic effect, is increased whereas the dose of the drug remaining in the upper airways (and potentially responsible for systemic side effects) is reduced.

These data lead to an equivalence between BDP in the extrafine HFA formulation and BDP in the traditional $\mathrm{CFC}$ formulation of 1:2.5 (Fig. 2) [11]. This means that a dose of $100 \mathrm{mcg}$ of BDP in extrafine HFA formulation is equivalent, in terms of efficacy, to $250 \mathrm{mcg}$ of BDP in traditional CFC formulation, with a significant lower risk of systemic side effects.

Some studies have been performed in order to demonstrate the efficacy of this new BDP/Formoterol combination. A first study was done in order to compare the new combination with the combination with BDP at higher doses in the traditional CFC formulation plus inhaled formoterol, administered with two different inhalers; a third group of patients was treated with BDP alone in the traditional CFC formulation [12]. The study shows that the new BDP/Formoterol combination was equivalent to the traditional extemporary combination with higher doses of BDP in terms of pulmonary function and rate of severe exacerbations, but it was better than comparators as regards the percentage of days without asthma symptoms or of days with asthma control (Fig. 3). Notably, morning serum cortisol levels increased significantly versus baseline with BDP/formoterol, but remained substantially unchanged with the other regimens, suggesting that the control of asthma could be maintained at least as well as with traditional higher doses of BDP with the potential for lower side effects.

Two similar studies have been performed in order to show the non inferiority of this new combination in comparison with the other ICS-LABA combinations already present in the market. The first study compared the new $\mathrm{BDP} /$ formoterol in the extrafine HFA formulation with budesonide/formoterol combination; the dose ratio of BDP: Budesonide was 1:2, which is different from the traditional equivalence reported in the equivalence table of ICS in several international guidelines [2, 8-9]. In this study, more than 200 moderate asthmatic subjects, uncontrolled under ICS at doses lower than $1000 \mathrm{mcg}$ of BDP or equivalent, were enrolled in a classic double blind randomised parallel group 3 month study [13]. The main outcome of the study was morning peak expiratory flow (PEF). In both groups, PEF significantly increased just after few weeks and remained higher than baseline during all the study period, without any significant difference between both groups (Fig. 4). The same efficacy was observed in the two groups as regards day-time and nocturnal symptoms, and the rate of asthma exacerbations. The second study used the same study design, comparing the 


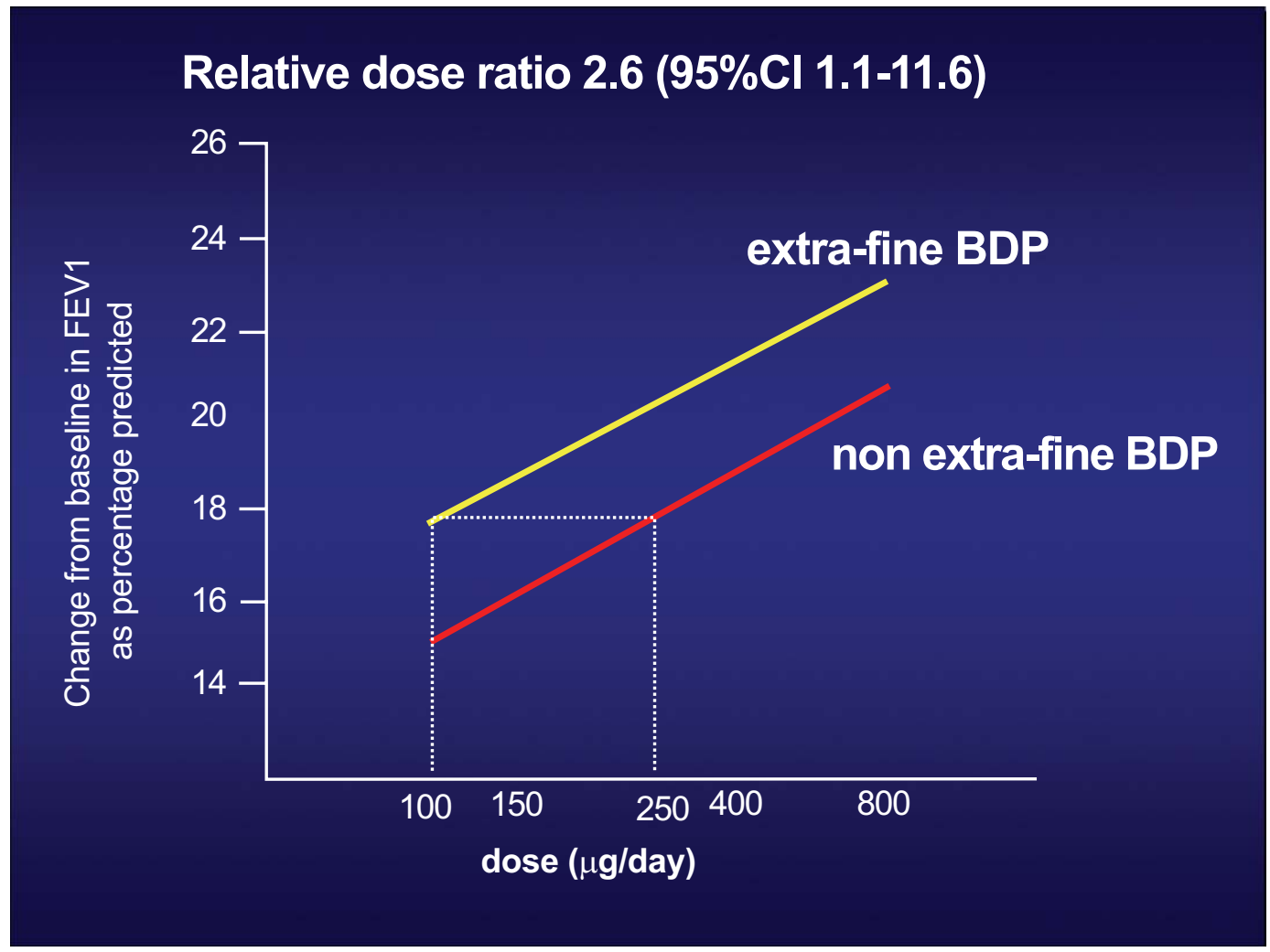

Fig. (2). Dose-effect ratio, in terms of FEV1 increase, of the two different formulations of beclomethasone: the traditional CFC formulation, and the new extra-fine HFA formulation. To obtain the same effect on FEV1 of $250 \mathrm{mcg}$ of BDP in the traditional formulation, only 100 mcg of BDP in the new extra-fine HFA formulation is required [11a, 11b].

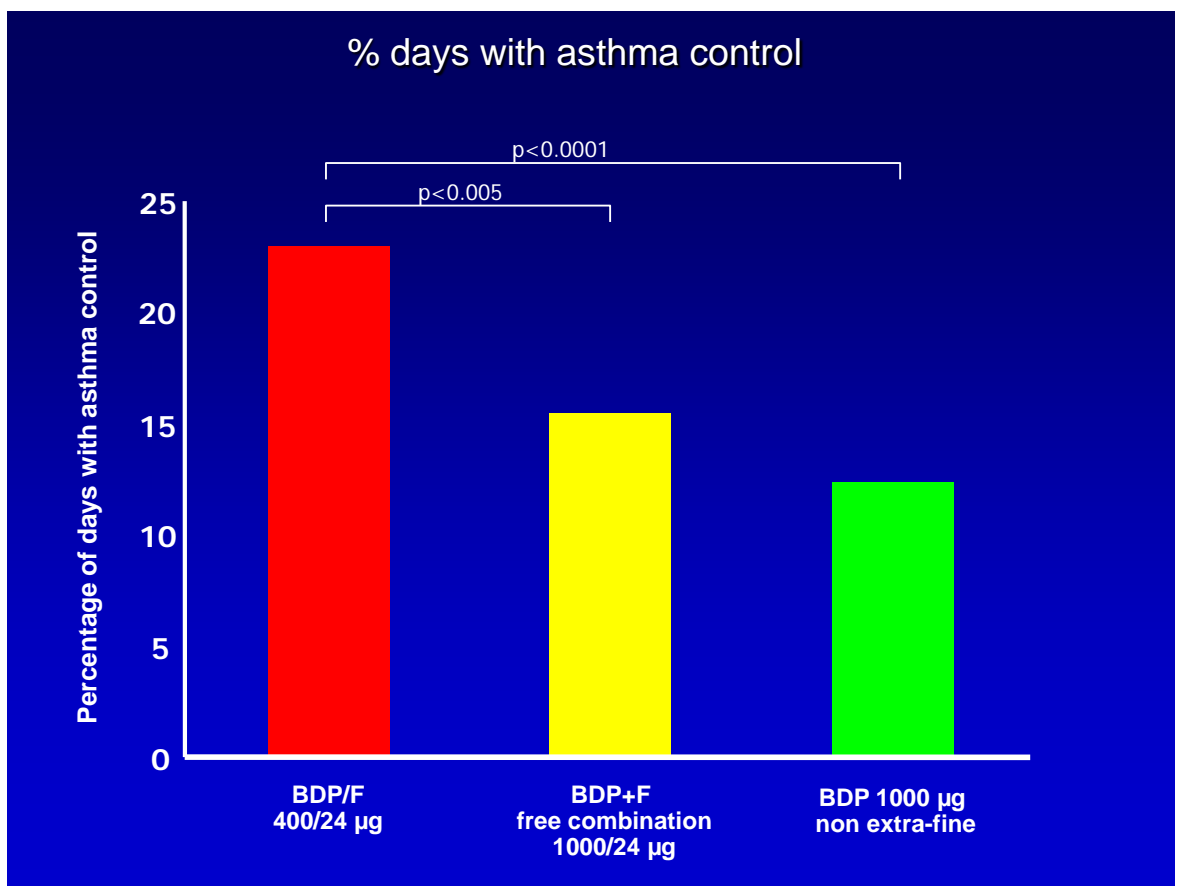

Fig. (3). Level of asthma control in patients treated with the new budesonide/formoterol extra-fine HFA formulation, the traditional combination by two distinct inhalers, or high dose beclomethasone alone [12].

new BDP/formoterol combination in extrafine HFA formulation with the fluticasone/salmeterol combination [14]. In this study, the dose ratio of BDP:fluticasone was 1:1.25, again different from the traditional equivalence reported in the equivalence table of ICS. The characteristics of the studied population was identical to the previous paper, as well as the main outcome (morning PEF). Also this study confirmed the equivalence between this new combination with the oldest fluticasone/salmeterol combination at different dose, in terms of pulmonary function, asthma symptoms and exacer- 


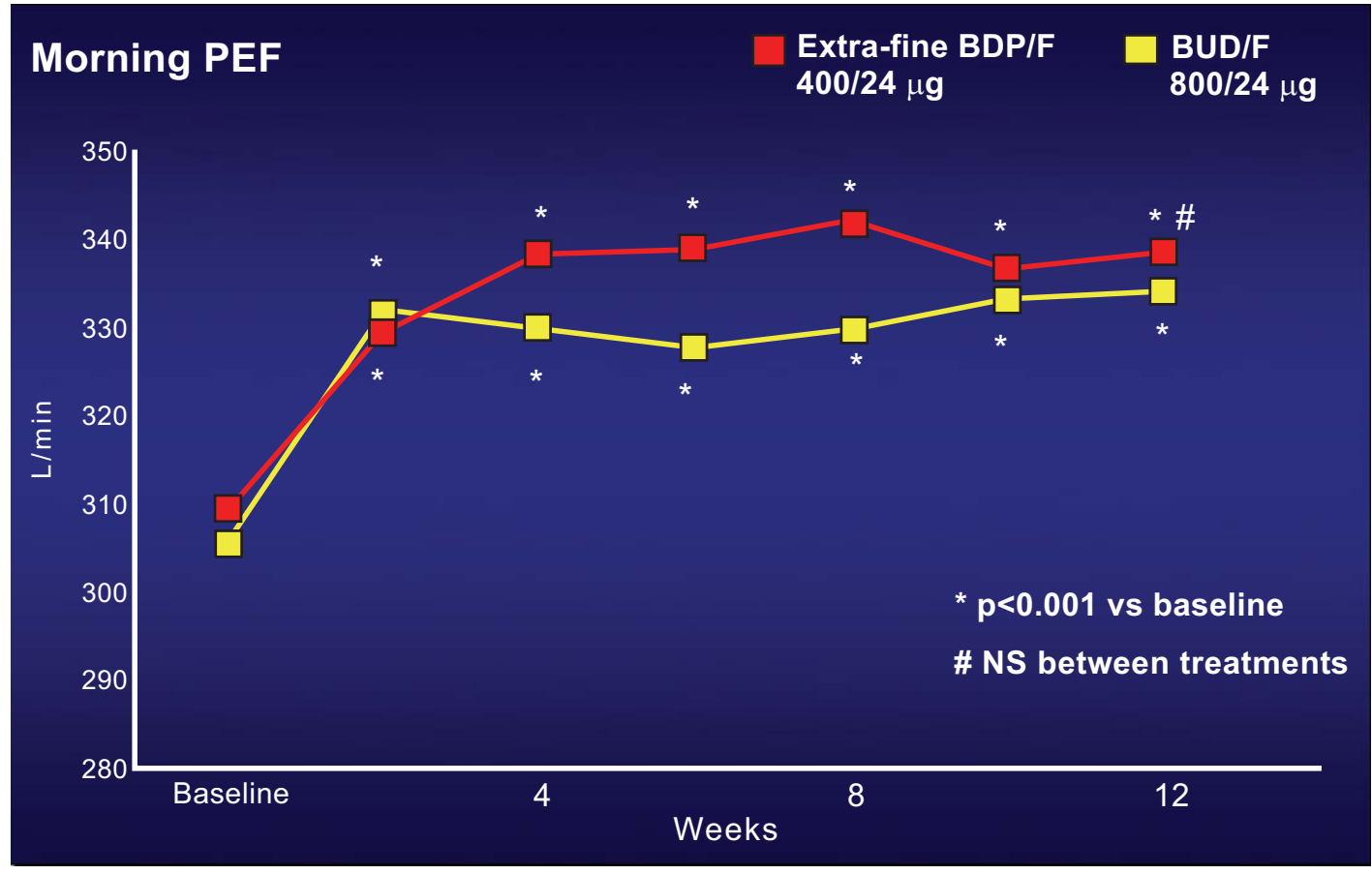

Fig. (4). Increase from baseline in the Peak Expiratory Flow (PEF) in subjects treated with the new beclomethasone/formoterol combination in extra-fine HFA formulation, in comparison with the traditional budesonide/formoterol combination. In both groups, PEF progressively increased, without any significant difference between two groups [13].

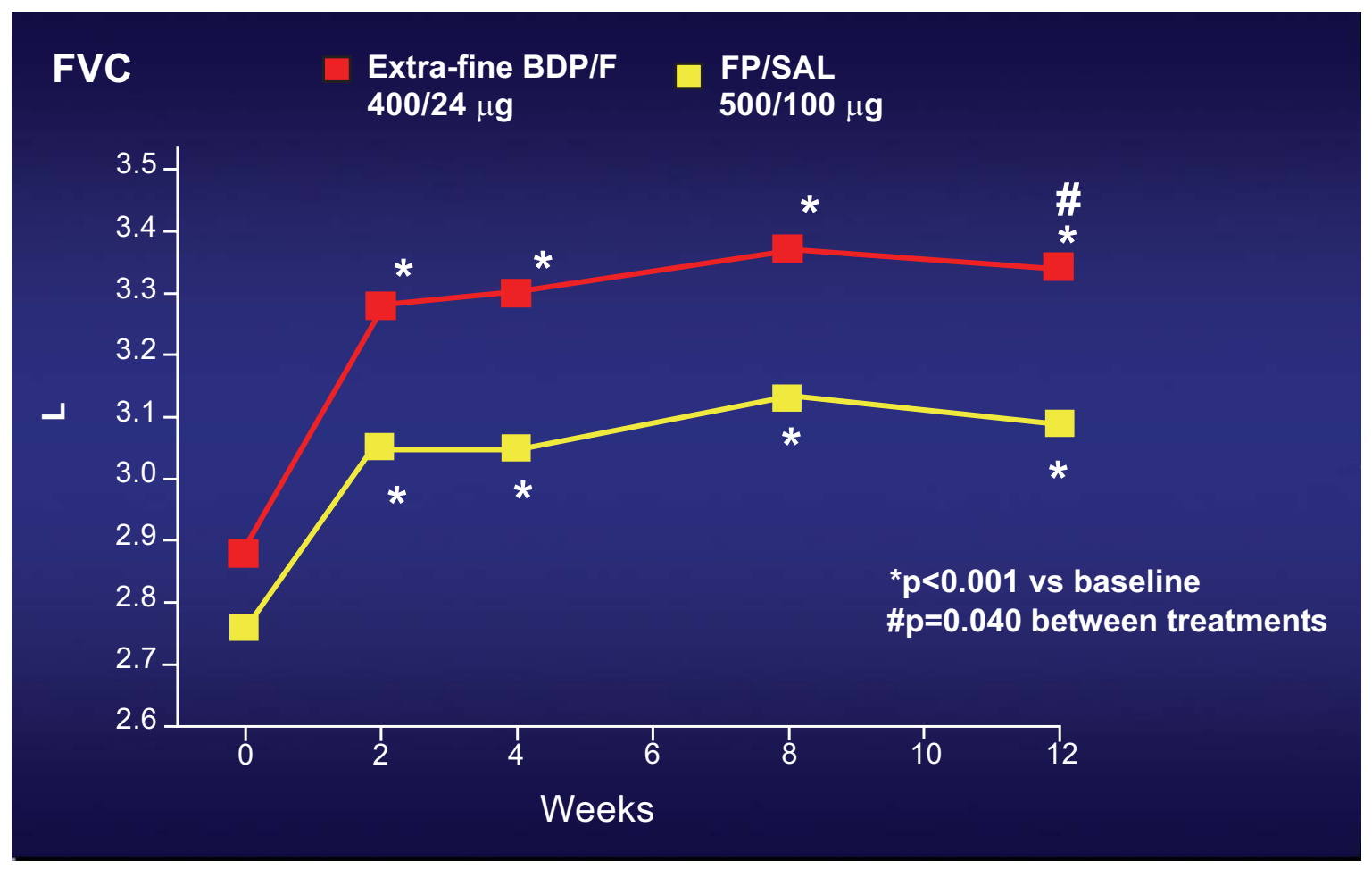

Fig. (5). Increase from baseline in the Forced Vital Capacity (FVC) in subjects treated with the new beclomethasone/formoterol combination in extra-fine HFA formulation, in comparison with the traditional fluticasone/salmeterol combination. In both groups, FVC progressively increased, but this increase was significantly better with the new beclomethasone/formoterol combination [16].

bations. Furthermore, a significant better improvement in forced vital capacity (FVC) was observed with the new $\mathrm{BDP} /$ formoterol combination in comparison with fluticasone/salmeterol combination (Fig. 5). This observation sug- gests that the efficacy on may be better with the new $\mathrm{BDP} /$ Formoterol in the extrafine HFA formulation, due to the fine particle size of this combination. 
Other studies are in progress with this new combination, both in asthma (including the efficacy of BDP/formoterol as "maintenance and reliever" strategy) and in COPD.

\section{CONCLUSIONS}

The main objective of the management of asthma is the clinical and functional control; this may be easily evaluated by an integrated measurement of the frequency of symptoms and rescue medication, the absence of limitation in daily life, the rate of exacerbations, and the level of pulmonary function. The combination between an inhaled corticosteroid and a long-acting-beta2-agonist is the preferred option in a large majority of asthmatic patients. Different pharmacologic strategies can be used in order to reach and maintain the control, allowing to tailor the therapeutic plan to the characteristics of the patients or the disease. The new beclomethasone/formoterol combination may increase the possibility to manage asthma, using low dose ICS with a great clinical efficacy and a low risk of systemic side effects.

\section{REFERENCES}

[1] Rabe KF, Vermeire PA, Soriano JB, Maier WC. Clinical management of asthma in 1999: the Asthma Insights and Reality in Europe (AIRE) study. Eur Respir J 2000; 16: 802-7.

[2] Global Initiative for Asthma. Up-date 2008. www.ginasthma.org

[3] Bateman ED, Boushey HA, Bousquet J, et al., GOAL Investigators Group. Can guideline-defined asthma control be achieved? The Gaining Optimal Asthma Control Study. Am J Respir Crit Care Med 2004; 170: 836-44.

[4] Bateman ED, Bousquet J, Keech ML, Busse WW, Clark TJ, Pedersen SE. The correlation between asthma control and health status: the GOAL study. Eur Respir J 2007; 29: 56-62.

[5] Ward C, Pais M, Bish R, et al. Airway inflammation, basament membrane thickning and bronchial hyperresponsiveness in asthma. Thorax 2002; 57: 309-17.

[6] Greenstone IR, Ni Chroinin MN, Masse V, et al. Combination of inhaled long-acting beta2-agonists and inhaled steroids versus higher dose of inhaled steroids in children and adults with persistent asthma. Cochrane Database Syst Rev 2005; (4): CD005533.
[7] Barnes PJ. Scientific rationale for inhaled combination therapy with long-acting beta2-agonists and corticosteroids. Eur Respir J 2002; 19: 182-91.

[8] British Thoracic Society Scottish Intercollegiate Guidelines Network. British guideline on the management of asthma. Thorax 2008; (Suppl 4): iv1-121.

[9] Becker A, Lemière C, Bérubé D, et al. Asthma Guidelines Working Group of the Canadian Network For Asthma Care. Summary of recommendations from the Canadian Asthma Consensus guidelines, 2003. CMAJ 2005; 173(6 Suppl): S3-11.

[10] Lasserson TJ, Cates CJ, Ferrara G, Casali L. Combination fluticasone and salmeterol versus fixed dose combination budesonide and formoterol for chronic asthma in adults and children. Cochrane Database Syst Rev 2008; (3): CD004106.

[11] (a) Rigamonti E, Kottakis I, Pelc M, et al. Comparison of a new extrafine beclomethasone dipropionate HFA134a-formulated pMDI with a standard BDP CFC pMDI in adults with moderate persistent asthma. 16 ${ }^{\text {th }}$ Annual Meeting ERS. Eur Respir J 2006; 28 (Suppl 50): 1236s. (b) Busse WW, Brazinsky S, Jacobson K, et al. Efficacy response of inhaled beclomethasone dipropionate in asthma is proportional to dose and is improved by formulation with a new propellant. J Allergy Clin Immunol 1999; 104: 215-22

[12] Huchon G, Magnussen H, Chuchalin A, Dymek L, Gonod FB, Bousquet J. Lung function and asthma control with beclomethasone and formoterol in a single inhaler. Respir Med 2009; 103: 419.

[13] Papi A, Paggiaro PL, Nicolini G, Vignola AM, Fabbri LM. Inhaled Combination Asthma Treatment versus SYmbicort (ICAT SY) Study Group. Beclomethasone/formoterol versus budesonide/formoterol combination therapy in asthma. Eur Respir J 2007; 29: 682-9.

[14] Papi A, Paggiaro P, Nicolini G, Vignola AM, Fabbri LM. ICAT SE study group. Beclomethasone/formoterol $v s$ fluticasone/salmeterol inhaled combination in moderate to severe asthma. Allergy 2007; 62: 1182-8.

[15] Paggiaro P, Nicolini G, Papi A. Extrafine beclomethasone dipropionate/formoterol hydrofluoroalkane-propelled inhaler in asthma. Expert Rev Resp Med 2008; 2: 161-6.

[16] Nicolini G, Scichilone N, Bizzi A, Papi A, Fabbri LM. Beclomethasone/formoterol fixed combination for the management of asthma: patient considerations. Ther Clin Risk Manag 2008:4(5) $855-64$. 